\title{
Erratum: Waveguide QED: Power spectra and correlations of two photons scattered off multiple distant qubits and a mirror [Phys. Rev. A 91, 053845 (2015)]
}

\author{
Yao-Lung L. Fang (方耀龍) and Harold U. Baranger \\ (Received 18 October 2017; published 13 November 2017)
}

DOI: 10.1103/PhysRevA.96.059904

We present two corrections to the original article. First, Fig. 14 should be replaced by the figure shown below. The result shown for $k_{0} a=20 \pi$ in Fig. 14(b) of the original paper is incorrect. As the incoming photons are in a plane-wave state at the qubit frequency, there is no coupling to the qubit. Thus the correlation function $g_{2}(t)$ must be $g_{2}(t)=1$ for all times in this case. In addition to our original method, we have obtained results for this case using an exact time-dependent calculation based on the finite-difference time-domain method [1]. The main point of this figure was and remains the striking difference between the results of a Markovian and a non-Markovian calculation as exemplified by the $k_{0} a=20.5 \pi$ case.

Second, we correct a typographical error in the expression for $s_{2}(\infty)$ in Eq. (C4): A factor of $1 / 2$ is missing. The correct result is

$$
s_{2}(\infty)=\frac{1}{2} \frac{\Gamma \Omega^{2} / 2}{\Gamma\left(\Gamma^{2} / 4+\delta \omega^{2}\right)+\Gamma \Omega^{2} / 2} .
$$

The other results reported in the paper remain intact.

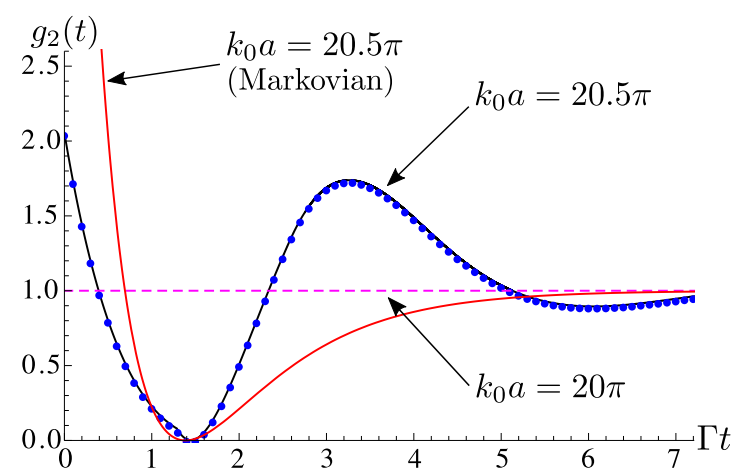

FIG. 14. Photon correlations $g_{2}(t)$ of a single qubit coupled to a semi-infinite waveguide with resonant incoming photons $\left(k=\omega_{0}\right)$ in the non-Markovian regime $\left(k_{0} a=20 \pi\right.$ and $\left.20.5 \pi\right)$. For $k_{0} a=20.5 \pi$, the full non-Markovian result calculated from scattering theory (the blue points) or the finite-difference time-domain [(FDTD) [1], the black curve] differs substantially from the Markovian result (the red curve) [as in Fig. 14(a)]. For $k_{0} a=20 \pi$, we check that the FDTD approach (the magenta dashed line) yields the exact result $g_{2}(t)=1$, which is obtained because the qubit decouples. [Parameters: The qubit frequency is $\omega_{0}=100 \Gamma$, and the FDTD step size is $\Delta=\lambda_{0} / 240$.]

[1] Y.-L. L. Fang, FDTD: solving 1+1D delay PDE, arXiv:1707.05943. 